\title{
Semi-Lagrangian relaxation applied to the uncapacitated facility location problem*
}

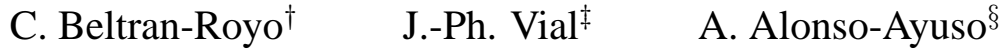

January 8, 2010

\begin{abstract}
We show how the performance of general purpose Mixed Integer Programming (MIP) solvers, can be enhanced by using the Semi-Lagrangian Relaxation (SLR) method. To illustrate this procedure we perform computational experiments on large-scale instances of the Uncapacitated Facility Location (UFL) problems with unknown optimal values. CPLEX solves 3 out of the 36 instances. By combining CPLEX with SLR, we manage to solve 18 out of the 36 instances and improve the best known lower bound for the other instances. The key point has been that, on average, the SLR approach, has reduced by more than $90 \%$ the total number of relevant UFL variables.
\end{abstract}

Keywords: Lagrangian relaxation, mixed integer programming, uncapacitated facility location (UFL) problem.

\section{Introduction}

Implementing an exact method for large scale MIP problems usually requires devising efficient cuts, efficient branching strategies, etc. to exploit the special structure of the problem at hand. In this paper we explore another possibility: to solve large scale MIP problems by combining the SLR approach with a general MIP solver. The idea is to take advantage of the steadily increasing power of general MIP codes. The aim of combining a general MIP solver with SLR is not to obtain the fastest procedure to solve a problem, but to enhance the performance of the MIP solver at low programming cost. Here we use the UFL problem, as a benchmark to illustrate the SLR approach. In order to obtain the best performance, one should use Erlenkotter type algorithms [14], which are especially designed for the UFL problem.

The Semi-Lagrangian Relaxation (SLR) method was introduced in [5] to solve the p-median problem. In order to solve a combinatorial problem, the SLR method has two main advantages

*This work has been partially supported by the Spanish government subsidy MTM2009-14039-C06-03 and by the 'Rey Juan Carlos University / Comunidad de Madrid' subsidy URJC-CM-2008-CET-3703.

${ }^{\dagger}$ Corresponding author, cesar.beltran@urjc.es, Statistics and Operations Research, Rey Juan Carlos University, Madrid, Spain.

${ }^{\ddagger}$ jpvial@ ordecsys.com, Professor Emeritus, University of Geneva (Switzerland) and Director of ORDECSYS, www.ordecsys.com.

§antonio.alonso@urjc.es, Statistics and Operations Research, Rey Juan Carlos University, Madrid, Spain. 
compared to the Lagrangian relaxation (LR): The SLR method closes the duality gap and gives an optimal integer solution as a byproduct. The disadvantage of the SLR method is that the relaxed problem is more difficult to solve than in the case of the LR.

SLR applies to problems with equality constraints. Like Lagrangian Relaxation (LR), the equality constraints are relaxed, but the definition of the semi-Lagrangian dual problem incorporates those constraints under the weaker form of inequalities. On combinatorial problems with positive coefficients, it has the strong property of achieving a zero duality gap. The method has been used with success to solve large instances of the p-median problem. In this paper we revisit the method and apply it to the celebrated Uncapacitated Facility Location (UFL) problem. We perform computational experiments on two main collections of UFL problems with unknown optimal values. CPLEX solves 3 out of the 36 instances. On one collection, we manage to solve to optimality 16 out of the 18 problems. On the second collection we solve 2 out of 18 instances. Nevertheless, we are able to improve the Lagrangian lower bound in this collection and thereby confirm that the Hybrid Multistart heuristic of [32] provides near optimal solutions (over 99\% optimal in most cases).

The UFL problem (also called the simple plant location problem) is a basic but important problem in location science [31]. See [29] for a survey. [27] and [24] improve the famous dualbased procedure of Donald Erlenkotter [14], where the condensed dual of the LP relaxation for the UFL problem is heuristically maximized by the coordinate ascent method. This procedure is followed by a Branch-and-Bound if necessary. [11] propose an projection method to improve the coordinate ascent method in order to compute an exact optimum of the condensed dual problem. [15] adapt Erlenkotter's method to effectively solve the so called two-echelon location problem, which generalizes the UFL problem.

On the other hand, after 1990 contributions to the UFL problem mainly dealt with heuristic methods. Some of the most successful methods are: Tabu search in [33, 16], a combination of Tabu search, Genetic Algorithm and Greedy Randomized Adaptative Search in [26] and Hybrid Multistart heuristic in [32]. Other contributions have investigated enhanced versions of the UFL problem, as the two-stage UFL [28] or have investigated new computational techniques applied to the UFL problem, as parallel interior point methods [12].

Lagrangian relaxation [20] has also been used to solve the UFL problem, though with less success than the LP dual-based procedures. For example [19] proposes to strengthen the Lagrangian relaxation (equivalent to the LP relaxation) by using Benders inequalities. From a more theoretical point of view, [30] studies the duality gap of the UFL problem. A complete study of valid inequalities, facets and lifting theorems for the UFL problem can be found in $[8,9,10]$.

This paper is organized as follows: In Section 2 we review the main properties of the SLR and discuss two algorithms to solve its associated dual problem: Proximal-ACCPM and the dual ascent algorithm. In Section 3, we apply the SLR to the UFL problem, develop some related theoretical properties and detail a specialization of the dual ascent algorithm for the UFL case. Section 4 reports our empirical study. Our conclusions are given in a last section.

\section{Semi-Lagrangian relaxation}

The concept of semi-Lagrangian relaxation was introduced in [5]. In this section, we summarize the main results obtained in that paper and simplify the proofs given there. 
Consider the problem, to be named "primal" henceforth.

$$
\begin{aligned}
z^{*}=\min _{x} & c^{T} x \\
\text { s.t. } & A x=b, \\
& x \in S \subset X \cap \mathbb{N}^{n} .
\end{aligned}
$$

Assumption 1 The components of $A \in \mathbb{R}^{m} \times \mathbb{R}^{n}, b \in \mathbb{R}^{m}$ and $c \in \mathbb{R}^{n}$ are nonnegative.

Assumption $2 X$ is a polyhedral set, $0 \in S$ and (1) is feasible.

Assumptions 1 and 2 together imply that (1) has an optimal solution.

The semi-Lagrangian relaxation consists in adding the inequality constraint $A x \leq b$ and relaxing $A x=b$ only. We thus obtain the dual problem

$$
\max _{u \in \mathbb{R}^{m}} \mathcal{L}(u)
$$

where $\mathcal{L}(u)$ is the semi-Lagrangian dual function defined as

$$
\begin{aligned}
\mathcal{L}(u)=\min _{x} & c^{T} x+u^{T}(b-A x) \\
\text { s.t. } & A x \leq b, \\
& x \in S .
\end{aligned}
$$

Note that with our assumptions the feasible set of (3) is bounded. We also have that $x=0$ is feasible to (3); hence (3) has an optimal solution. $\mathcal{L}(u)$ is well-defined, but the minimizer in (3) is not necessarily unique. With some abuse of notation, we write

$$
x(u)=\arg \min _{x}\left\{c^{T} x+u^{T}(b-A x) \mid A x \leq b, x \in S\right\}
$$

to denote one such minimizer. With this notation we may write $\mathcal{L}(u)=\left(c-A^{T} u\right)^{T} x(u)+b^{T} u$. We denote $U^{*}$ the set of optimal solutions of problem (2). Finally, given two sets $A$ and $B$, its addition corresponds to $A+B=\{a+b: a \in A$ and $b \in B\}$.

Theorem 1 The following statements hold [5].

1. $\mathcal{L}(u)$ is concave and $b-A x(u)$ is a subgradient at $u$.

2. $\mathcal{L}(u)$ is monotone and $\mathcal{L}\left(u^{\prime}\right) \geq \mathcal{L}(u)$ if $u^{\prime} \geq u$, with strict inequality if $u^{\prime}>u$ and $u^{\prime} \notin U^{*}$.

3. $U^{*}+\mathbb{R}_{+}^{m}=U^{*}$; thus $U^{*}$ is an unbounded (convex) set.

4. If $x(u)$ is such that $A x(u)=b$, then $u \in U^{*}$ and $x(u)$ is optimal for problem (1).

5. Conversely, if $u \in \operatorname{int}\left(U^{*}\right)$, then any minimizer $x(u)$ is optimal for problem (1).

6. The SLR closes the duality gap for problem (1). 
Now, we simplify the proof given in [5].

Proof: From the definition of the SLR function as a pointwise minimum, the inequality

$$
\begin{aligned}
\mathcal{L}\left(u^{\prime}\right) & \leq c^{T} x(u)+\left(u^{\prime}\right)^{T}(b-A x(u)) \\
& =\mathcal{L}(u)+(b-A x(u))^{T}\left(u^{\prime}-u\right),
\end{aligned}
$$

holds for any pair $u, u^{\prime}$. This shows that $\mathcal{L}(u)$ is concave and that $(b-A x(u))$ is a subgradient at $u$. This proves statement 1 .

To prove statement 2 , we note, in view of $b-A x\left(u^{\prime}\right) \geq 0$ and $u^{\prime} \geq u$, that we have the chain of inequalities

$$
\begin{aligned}
\mathcal{L}\left(u^{\prime}\right) & =c^{T} x\left(u^{\prime}\right)+\left(b-A x\left(u^{\prime}\right)\right)^{T} u^{\prime}, \\
& =c^{T} x\left(u^{\prime}\right)+\left(b-A x\left(u^{\prime}\right)\right)^{T} u+\left(b-A x\left(u^{\prime}\right)\right)^{T}\left(u^{\prime}-u\right), \\
& \geq c^{T} x\left(u^{\prime}\right)+\left(b-A x\left(u^{\prime}\right)\right)^{T} u, \\
& \geq c^{T} x(u)+(b-A x(u))^{T} u=\mathcal{L}(u) .
\end{aligned}
$$

This proves the first part of the third statement. If $u^{\prime} \notin U^{*}$, then $0 \notin \partial \mathcal{L}\left(u^{\prime}\right)$, the subdifferential of $\mathcal{L}$ at $u^{\prime}$ ([22]), and one has $\left(b-A x\left(u^{\prime}\right)\right)_{j}>0$ for some $j$. Thus, $u<u^{\prime}$ implies $\left(b-A x\left(u^{\prime}\right)\right)^{T}\left(u^{\prime}-u\right)>0$. Hence, $\mathcal{L}\left(u^{\prime}\right)>\mathcal{L}(u)$.

The third statement is an immediate consequence of the monotone property of $\mathcal{L}(u)$. Furthermore, $U^{*}$ is convex since it is the optimal set of a concave function [22].

To prove the fourth statement, we note that $A x(u)=b$ implies $0 \in \partial \mathcal{L}(u)$, a necessary and sufficient condition of optimality for problem (2). Hence $u \in U^{*}$. Finally, since $x(u)$ is feasible to (1) and optimal for the relaxation (3) of problem (1), it is also optimal for (1).

To prove the fifth statement, assume now $u \in \operatorname{int}\left(U^{*}\right)$. In this case there exists $u^{\prime} \in U^{*}$ such that $u^{\prime}<u$; thus $(b-A x(u))^{T}\left(u-u^{\prime}\right) \geq 0$, with strict inequality if $b-A x(u) \neq 0$. In view of (5),

$$
0 \geq(b-A x(u))^{T}\left(u^{\prime}-u\right) \geq \mathcal{L}\left(u^{\prime}\right)-\mathcal{L}(u) .
$$

Thus $A x(u)=b$, and $x(u)$ is optimal to (1). It follows that the original problem (1) and the semi-Lagrangian dual problem (2) have the same optimal value (the last statement).

To close this Section we present the two methods we use to solve the SLR dual problem (2): The proximal analytic center cutting plane method (proximal-ACCPM) and dual ascent method. The first one consists in choosing a theoretically and practically efficient general method for solving the semi-Lagrangian dual problem. As for the p-median problem [5] we select the proximal analytic center cutting plane method (proximal-ACCPM). This ensures a small number of iterations, and seems to keep the oracle subproblem simple enough during the solution process. The other method is a variant of the dual ascent method (e.g., [6]) based on finite increases of the components of $u$. In fact, the dual ascent algorithm we use is in essence the dual multi-ascent procedure used in [27] to solve the Erlenkotter's 'condensed' dual of the UFL problem [14]. In the case of LR, the dual multi-ascent method does not necessarily converge. In the case of the SLR we prove finite convergence. 


\subsection{The proximal analytic center cutting plane method}

Function $\mathcal{L}(u)$ in problem (2) is by construction, concave and nonsmooth (it is implicitly defined as the pointwise minimum of linear functions in $u$ ). Extensive numerical experience shows that Proximal-ACCPM, is an efficient tool for solving (2). See, for instance, [18] and references therein included; see also [5] for experiments with the $p$-median problem.

In the cutting plane procedure, we consider a sequence of points $\left\{u^{k}\right\}_{k \in K}$ in the domain of $\mathcal{L}(u)$. We consider the linear approximation to $\mathcal{L}(u)$ at $u^{k}$, given by

$$
\mathcal{L}^{k}(u)=\mathcal{L}\left(u^{k}\right)+s^{k} \cdot\left(u-u^{k}\right)
$$

and have

$$
\mathcal{L}(u) \leq \mathcal{L}^{k}(u)
$$

for all $u$.

The point $u^{k}$ is referred to as a query point, and the procedure to compute the objective value and subgradient at a query point is called an oracle. Furthermore, the hyperplane that approximates the objective function $\mathcal{L}(u)$ at a feasible query point and defined by the equation $z=\mathcal{L}^{k}(u)$, is referred to as an optimality cut.

A lower bound to the maximum value of $\mathcal{L}(u)$ is provided by:

$$
\theta_{l}=\max _{k} \mathcal{L}\left(u^{k}\right)
$$

The localization set is defined as

$$
L_{K}=\left\{(u, z) \in \mathbb{R}^{n+1} \mid u \in \mathbb{R}^{n}, \quad z \leq \mathcal{L}^{k}(u) \quad \forall k \leq K, \quad z \geq \theta_{l}\right\},
$$

where $K$ is the current iteration number. The basic iteration of a cutting plane method can be summarized as follows:

1. Select $(\bar{u}, \bar{z})$ in the localization set $L_{K}$.

2. Call the oracle at $\bar{u}$. The oracle returns one or several optimality cuts and a new lower bound $\mathcal{L}(\bar{u})$.

3. Update the bounds:

(a) $\theta_{l} \leftarrow \max \left\{\mathcal{L}(\bar{u}), \theta_{l}\right\}$.

(b) Compute an upper bound $\theta_{u}$ to the optimum of problem (2).

4. Update the lower bound $\theta_{l}$ and add the new cuts in the definition of the localization set (6).

These steps are repeated until a point is found such that $\theta_{u}-\theta_{l}$ falls below a prescribed optimality tolerance. The first step in the above algorithm sketch is not completely defined. Actually, cutting plane methods essentially differ in the way one chooses the query point. For instance, the intuitive choice of the Kelley point $(\bar{u}, \bar{z})$ that maximizes $z$ in the localization set [25] may prove disastrous, because it over-emphasizes the global approximation property of the localization set. Safer methods, as for example bundle methods [21] or Proximal-ACCPM 
$[17,18,13,2]$, introduce a regularizing scheme to avoid selecting points too "far away" from the best recorded point. In this paper we use Proximal-ACCPM (Proximal Analytic Center Cutting Plane Method) which selects the proximal analytic center of the localization set. Formally, the proximal analytic center is the point $(\bar{u}, \bar{z})$ that minimizes the logarithmic barrier function of the localization set plus a quadratic proximal term which ensures the existence of a unique minimizer. This point is relatively easy to compute using the standard artillery of Interior Point Methods. Furthermore, Proximal-ACCPM is robust, efficient and particularly useful when the oracle is computationally costly —as is the case in this application.

\subsection{A dual ascent method}

We state the algorithm first and then prove finite convergence. This algorithm, at each iteration, increases some or all of the coordinates of the dual iterate $u^{k}$, by at least $\Delta>0$.

\section{Algorithm 1: Dual ascent algorithm (basic iteration).}

1. Solve the oracle: Compute

$$
x^{k}=\arg \min _{x}\left\{c^{T} x+\left(u^{k}\right)^{T}(b-A x) \mid A x \leq b, x \in S\right\},
$$

where $u^{k}$ is the current dual iterate.

2. Stopping criterion: If $s^{k}:=b-A x^{k}=0$, stop. $\left(x^{k}, u^{k}\right)$ is an optimal primal-dual point.

3. Update the dual iterate: For $j=1, \ldots, n$, set

$$
u_{j}^{k+1}= \begin{cases}u_{j}^{k}+\delta_{j}^{k} & \text { if } s_{j}^{k}>0, \\ u_{j}^{k} & \text { otherwise }\end{cases}
$$

where $\delta_{j}^{k} \geq \Delta$.

We now prove finite convergence of the above algorithm.

\section{Theorem 2 The following statements hold.}

1. Algorithm 1 is a dual ascent method when applied to solve the SLR dual problem: for any two consecutive iterates $u^{k}$ and $u^{k+1}$ we have $\mathcal{L}\left(u^{k+1}\right)>\mathcal{L}\left(u^{k}\right)$.

2. Let us suppose that $u^{0} \geq 0$ and that $U^{*} \neq \emptyset$. Algorithm 1 converges to an optimal dual point $u \in U^{*}$ after finitely many iterations.

Proof: Let us prove statement 1 first. The updating procedure of Algorithm 1 consists in increasing some components of the current dual point $u^{k}$ (step 3). Thus, $u^{k+1}>u^{k}$ and by Theorem 1.2 we have that $\mathcal{L}\left(u^{k+1}\right)>\mathcal{L}\left(u^{k}\right)$.

The proof of statement 2 goes as follows. Let us consider the sequence $\left\{s^{k}\right\}$ of subgradients generated by the algorithm. We have two exclusive cases.

Case 1: There exists $k_{0}$ such that $s^{k_{0}}=0$. Then $0 \in \partial \mathcal{L}\left(u^{k_{0}}\right)$ and $u^{k_{0}} \in U^{*}$. 
Case 2: At least for one component of $s^{k}$, say the 1st, there exists a subsequence $\left\{s_{1}^{k_{i}}\right\} \subset$ $\left\{s_{1}^{k}\right\}$ such that $s_{1}^{k_{i}} \neq 0$ for all $i=0,1,2, \ldots$. We will prove by contradiction that this case cannot happen.

By definition of the algorithm we will have:

$$
u_{1}^{k_{i}} \geq u_{1}^{k_{0}}+i \Delta
$$

Let us show that the subsequence $\left\{\mathcal{L}\left(u^{k_{i}}\right)\right\}$ is unbounded from above which contradicts the hypothesis $U^{*} \neq \emptyset$. Let us define $J^{k_{i}}=\left\{j \mid s_{j}^{k_{i}}>0\right\}$. Since $x$ is a binary vector, it implies, by Assumption 1, that there exists an absolute constant $\eta>0$ such that

$$
\min _{j} \min _{x}\left\{s_{j}=(b-A x)_{j} \mid(b-A x)_{j}>0\right\}=\eta .
$$

Thus $s_{j}^{k_{i}} \geq \eta$ for all $j \in J^{k_{i}}$ and for all $i$.

Using the fact that $c^{T} x \geq 0$ and that $u^{k_{i}} \geq 0$, we have

$$
\begin{aligned}
\mathcal{L}\left(u^{k_{i}}\right) & =c^{T} x\left(u^{k_{i}}\right)+\left(b-A x\left(u^{k_{i}}\right)\right)^{T} u^{k_{i}} \\
& =c^{T} x\left(u^{k_{i}}\right)+\left(s^{k_{i}}\right)^{T} u^{k_{i}} \\
& \geq\left(s^{k_{i}}\right)^{T} u^{k_{i}} \\
& =\sum_{j \in J^{k_{i}}} s_{j}^{k_{i}} u_{j}^{k_{i}} \\
& \geq u_{1}^{k_{i}} \eta \\
& \geq\left(u_{1}^{k_{0}}+i \Delta\right) \eta .
\end{aligned}
$$

Thus $\lim _{i \rightarrow \infty} \mathcal{L}\left(u^{k_{i}}\right)=+\infty$.

\section{SLR applied to the UFL problem}

In the Uncapacitated Facility Location (UFL) problem we have a set of 'facilities' indexed by $I=\{1, \ldots, m\}$ and a set of 'clients' indexed by $J=\{1, \ldots, n\}$. The purpose is to open facilities relative to the set of clients, and to assign each client to a single facility. The cost of an assignment is the sum of the shortest distances $c_{i j}$ from a client to a facility plus the fixed costs of opened facilities $f_{i}$. The distance is sometimes weighed by an appropriate factor, e.g., the demand at a client node. The objective is to minimize this sum. The UFL problem is NP-hard [29] and can be formulated as follows.

$$
\begin{array}{rl}
z^{*}=\min _{x, y} & z(x, y) \\
\text { s.t. } & \sum_{i=1}^{m} x_{i j}=1, \quad j \in J, \\
& x_{i j} \leq y_{i}, \quad i \in I, j \in J, \\
& x_{i j}, y_{i} \in\{0,1\},
\end{array}
$$


where

$$
z(x, y)=\sum_{i=1}^{m} \sum_{j=1}^{n} c_{i j} x_{i j}+\sum_{i=1}^{m} f_{i} y_{i} .
$$

$x_{i j}=1$ if facility $i$ serves the client $j$, otherwise $x_{i j}=0$ and $y_{i}=1$ if we open facility $i$, otherwise $y_{i}=0$.

Following the ideas of the preceding section, we formulate the semi-Lagrangian relaxation of the UFL problem. We obtain the dual problem

$$
\max _{u \in \mathbb{R}^{n}} \mathcal{L}(u)
$$

and the oracle (note that, now, we keep the equality constraint (8b) as an inequality)

$$
\begin{array}{rl}
\mathcal{L}(u)=\min _{x, y} & f(u, x, y) \\
\text { s.t. } \quad & \sum_{i=1}^{m} x_{i j} \leq 1,, \quad j \in J, \\
& x_{i j} \leq y_{i}, \quad i \in I, j \in J, \\
& x_{i j}, y_{i} \in\{0,1\},
\end{array}
$$

where

$$
\begin{aligned}
f(u, x, y) & =\sum_{i=1}^{m} \sum_{j=1}^{n} c_{i j} x_{i j}+\sum_{i=1}^{m} f_{i} y_{i}+\sum_{j=1}^{n} u_{j}\left(1-\sum_{i=1}^{m} x_{i j}\right) \\
& =\sum_{i=1}^{m} \sum_{j=1}^{n}\left(c_{i j}-u_{j}\right) x_{i j}+\sum_{i=1}^{m} f_{i} y_{i}+\sum_{j=1}^{n} u_{j} .
\end{aligned}
$$

As in the previous section, we denote $(x(u), y(u))$ an optimal point for the oracle (11). The associated Lagrangian relaxation, corresponds to formulation (11) without constraints (11b).

\subsection{Properties of the SLR dual problem}

In this section we show that, in order to solve the SLR dual problem, we can restrict our dual search to a box. To this end, we define for each client $j$, its best combined costs as $\tilde{c}_{j}:=$ $\min _{i}\left\{c_{i j}+f_{i}\right\}$. The vector of best combined costs is thus $\tilde{c}:=\left(\tilde{c}_{1}, \ldots, \tilde{c}_{n}\right)$. Furthermore, for each client $j$, we sort its costs $c_{i j}$, and get the sorted costs

$$
c_{j}^{1} \leq c_{j}^{2} \leq \ldots \leq c_{j}^{m} .
$$

Theorem $3 u \geq \tilde{c} \Rightarrow u \in U^{*}$ and $u>\tilde{c} \Rightarrow u \in \operatorname{int}\left(U^{*}\right)$.

Proof: Consider the oracle

$$
\begin{aligned}
\min _{x} & \sum_{i=1}^{m}\left(f_{i} y_{i}+\sum_{j=1}^{n}\left(c_{i j}-u_{j}\right) x_{i j}\right)+\sum_{j=1}^{n} u_{j} \\
& \sum_{i=1}^{m} x_{i j} \leq 1, \forall j \\
& x_{i j} \leq y_{i}, \forall i, j \\
& x_{i j}, y_{i} \in\{0,1\} .
\end{aligned}
$$


Assume $u \geq \tilde{c}$. If there exists an optimal solution of the oracle such that $\sum_{i} x_{i j}=1, \forall j$, then, by Theorem 1, this solution is optimal for the original problem. Assume we have an oracle solution with $\sum_{i} x_{i j}=0$ for some $j$. Let $i_{k}$ be such that $\tilde{c}_{j}=f_{i_{k}}+c_{i_{k} j}$. By hypothesis, $\tilde{c}_{j}-u_{j} \leq 0$. Thus, $f_{i_{k}}+\left(c_{i_{k} j}-u_{j}\right) \leq 0$ and one can set $x_{i_{k} j}=1$ and $y_{i_{k}}=1$ without increasing the objective value. The modified solution is also optimal. Hence, there exists an optimal oracle solution with $\sum_{i} x_{i j}=1, \forall j$ and $u \in U^{*}$. The second statement of the theorem follows from $\tilde{c} \in U^{*}$ and statement 3 of Theorem 1 .

Theorem 4 If $u \in \operatorname{int}\left(U^{*}\right)$, then $u \geq c^{1}$.

Proof: Let us assume assume that $u_{j_{0}}<c_{j_{0}}^{1}$ for some $j_{0} \in J$ and see that this contradicts $u \in \operatorname{int}\left(U^{*}\right)$. If $u_{j_{0}}<c_{j_{0}}^{1}$ then $c_{j_{0}}^{k}-u_{j_{0}}>0$ for all $k \in I$. Any optimal solution $x(u)$ is such that $x_{i j_{0}}(u)=0$, for all $i \in I$. Hence, $1-\sum_{i \in I} x_{i j_{0}}(u)=1$ and by Theorem $1, u$ is not in $\operatorname{int}\left(U^{*}\right)$.

Corollary 1 Let us consider the scalar $\epsilon>0$, the vector $\bar{\epsilon}$, where each component is equal to $\epsilon$, and the box

$$
\mathcal{B}:=\left\{u \in \mathbb{R}^{n} \mid c^{1}<u \leq \tilde{c}+\bar{\epsilon}\right\} .
$$

Then, for the UFL problem one has

$$
\operatorname{int}\left(U^{*}\right) \cap \mathcal{B} \neq \emptyset
$$

This corollary implies that the search for an optimal dual point can be confined to a box. In particular, taking $\bar{u}=\tilde{c}+\bar{\epsilon}$ and solving the oracle for $\bar{u}$, yields a primal optimal solution in one step. As pointed out earlier, it is likely to be impractical because the oracle is too difficult at that $\bar{u}$. It is also, likely that there is a smaller $u^{*} \in U^{*}$, for which the oracle subproblem would be easier (with less binary variables) and hopefully tractable by an integer programming solver. This justifies the use of dual methods that increase the dual iterates in small steps.

\subsection{Structure of the oracle: the core subproblem}

In this section, we study the structure of the UFL oracle as a function of the dual variable $u$. We shall show how some primal variables $x_{i j}$ can be set to zero when $u_{j}$ is small enough. This operation, which reduces the size of the oracle, is quite common in Lagrangian relaxation applied to combinatorial optimization. There, using some appropriate argument, one fixes some variables of the oracle and obtains a reduced-size oracle called the core subproblem. (See, for instance, $[7,1]$.)

We now define the core UFL subproblem in the SLR case. Let $c(u)$ be the matrix of reduced costs such that $c(u)_{i j}=c_{i j}-u_{j}$. Let $G=(V \times W, E)$ be the bipartite graph associated to the UFL problem, such that each node in $V(W)$ represents a facility (client) and the edge $e_{i j}$ exists if facility $i$ can serve client $j . c(u)_{i j}$ is thus the reduced cost associated to $e_{i j}$. Let $E(u) \subset E$ be the subset of edges with strictly negative reduced cost for a given dual point $u$. Let $V(u) \subset V$ and $W(u) \subset W$ be the adjacent vertices to $E(u)$. Let $G(u)=(V(u) \times W(u), E(u))$ be the induced bipartite subgraph. We call $G(u)$ the core graph. 
It is easy to see that for any $c(u)_{i j} \geq 0$ there exists $x(u)$ such that $x(u)_{i j}=0$. Therefore, we can restrict our search to the core graph $G(u)$ to compute $x(u)$. The advantage of solving oracle (11) with core graph $G(u)$ is that in $G(u)$ we may have much less edges (variables $x_{i j}$ ) and facility nodes (variables $y_{j}$ ), as we will see in Section 4.6. A further advantage of solving the core subproblem is that, even though $G$ is usually a connected graph, $G(u)$ may be decomposable into independent subgraphs and then we can decompose the core subproblem into smaller (easier) subproblems.

We now study the inverse image of the mapping $G(u)$. In other words, we wish to describe the set of $\bar{u}$ 's that have the graph $\bar{G}$ as a common image through the mapping $G(u)$. It is easy to see that it is a simple box.

To define an elementary box, we use the sorted costs to partition the domain of each coordinate $u_{j}$ into intervals of the form $\left.] c_{j}^{k}, c_{j}^{k+1}\right]$, with the convention that $c_{j}^{m+1}=+\infty$. Note that some boxes may be empty (if $c_{j}^{k}=c_{j}^{k+1}$ ). These coordinate partitions induce a partition of the box $\mathcal{B}$ into elementary boxes. It is not difficult to show that, there is a bijection between core graphs $G(u)$ and elementary boxes. Therefore, it is enough to restrict the dual search to one representative point per elementary box. The dual ascent algorithm to be presented in the next section will exploit this structure.

\subsection{The dual ascent algorithm to solve the SLR dual problem}

We now present a specialized version of the dual ascent method (Algorithm 1) for the UFL problem. We know by Corollary 1 , that the dual search can be confined to the box $\mathcal{B}$. The discussion of the previous section shows that $\mathcal{B}$ can be partitioned into elementary boxes and that it is enough to restrict the dual search to one representative point per elementary box. Our choice is to take this representative point, as small as possible within each elementary box. Since an elementary box is determined by the intervals $] c_{j}^{k}, c_{j}^{k+1}$, we choose the representative point to be $u_{j}=c_{j}^{l(j)}+\epsilon \quad(j=1, \ldots, n)$, for some fixed $\epsilon>0$.

Our algorithmic scheme is as follows: Take a small $\epsilon>0$ and for each client $j$, take some $l(j) \in I$ (see Section 4.1 for details). Start the dual search at $u_{j}^{0}:=c_{j}^{l(j)}+\epsilon$ (each client node will have exactly $l(j)$ core edges). Query the oracle, and if the current dual iterate is not optimal, update it. To maintain the number of core edges as low as possible, at each iteration, we add at most one edge per client, that is, we update $u_{j}$ from $c_{j}^{l(j)}+\epsilon$ to $c_{j}^{l(j)+1}+\epsilon$, for some $l(j) \in I$. We only update the coordinates of the dual iterate $u^{k}$ whose corresponding subgradient coordinate is not null. The details of this simple procedure are as follows.

\section{Algorithm 2: Dual ascent algorithm (UFL case).}

1. Initialization: Set $k=0$ and $\epsilon>0$. For each client $j \in J=\{1, \ldots, n\}$, set

(a) $u_{j}^{0}=c_{j}^{l(j)}+\epsilon$ for some $l(j) \in I=\{1, \ldots, m\}$,

(b) $\tilde{c}_{j}=\min _{i}\left\{c_{i j}+f_{i}\right\}$,

(c) $c_{j}^{m+1}=+\infty$. 
2. Oracle call: Compute $\mathcal{L}\left(u^{k}\right),\left(x\left(u^{k}\right), y\left(u^{k}\right)\right)$ and $s^{k}$ where

$$
s_{j}^{k}=1-\sum_{i=1}^{m} x_{i j}^{k},
$$

for all $j \in J$. (Note that $s_{j}^{k} \in\{0,1\}$.)

3. Stopping criterion: If $s^{k}=0$ then stop. The pair $\left(u^{k} ;\left(x\left(u^{k}\right), y\left(u^{k}\right)\right)\right.$ is a primal-dual optimal point.

4. Multiplier updating: For each $j \in J$ such that $s_{j}^{k}=1$, set

$$
u_{j}^{k+1}=\min \left\{c_{j}^{l(j)+1}, \tilde{c}_{j}\right\}+\epsilon \text { and } l(j)=\min \{l(j)+1, n\} .
$$

5. Set $k=k+1$ and go to Step 2 .

By construction, the iterates are monotonic with at least one strictly increasing coordinate. The algorithm converges in a finite number of iterations. The semi-Lagrangian dual function is also monotonic, which makes the algorithm a dual-ascent.

\section{Computational experiments}

The objective of our numerical test is twofold: first, we study the quality of the solution given by the semi-Lagrangian relaxation (SLR) and second we study the SLR performance in terms of CPU time. The CPU time limit is set to 7200 seconds. First, we compare our combined SLRCPLEX approach to the plain CPLEX. Second, we compare our results to the results reported in [32] and in the Uncapacitated Facility Location Library (UflLib) [23].

\subsection{Parameter setting for the algorithms}

Programs have been written in MATLAB 7.0 and run on a PC (Pentium-IV, 3.0 GHz, with 3 GB of RAM memory) under the Windows XP operating system. The reader should bear in mind that results reported in [32] were found on a different machine (SGI Challenge with 28 196-MHz MIPS R10000 processors, although each execution was limited to a single processor) and programs were implemented in $\mathrm{C}++$.

In our context and using the terminology of oracle based optimization [2], to call or to solve an oracle means to compute $\mathcal{L}\left(u^{k}\right)$ : Oracle 1 for the Lagrangian relaxation and Oracle 2 for the semi-Lagrangian relaxation. To solve Oracle 2 (a large-scale mixed integer program) we have intensively used CPLEX 9.1 (default settings) interfaced with MATLAB [3].

The dual ascent algorithm is implemented as stated in the previous section without parameter tuning $\left(\epsilon=10^{-3}\right)$. Proximal-ACCPM is used in its generic form as described in [2] with some tuning. We use Proximal-ACCPM in a two phase scheme: in the first phase we solve the Lagrangian relaxation (LR) dual problem. In the second phase we solve the semi-Lagrangian relaxation (SLR) dual problem. The Proximal-ACCPM parameter setting is as follows: In the LR phase, we use a proximal weight $\rho$ that at each Proximal-ACCPM iteration is updated within the range $\left[10^{-6}, 10^{4}\right]$ and the required precision is $10^{-6}$. In the SLR phase, we choose to fix 
the reference proximal point at the initial point $u^{0}$. As pointed out in [5], an optimal LR dual point $u_{L R}^{*}$ is a good starting point for the SLR. To be more precise, in our UFL computational experiments, for each client $j$, we take the $c_{j}^{l(j)}$ which is closest to $u_{L R j}^{*}$ and define $u_{j}^{0}:=$ $c_{j}^{l(j)}+\epsilon$, for some small $\epsilon>0$ (as we saw in Algorithm 2). The proximal weight $\rho$ has been fixed, by tuning, to $10^{-4}$ for the Barahona-Chudak instances and to $10^{-3}$ for the Koerkel-Ghosh instances.

\subsection{Instance description}

For our test we use 36 unsolved UFL instances that can be obtained in the UFL library UflLib (see Table 1). The first set, with 18 instances, is called Barahona-Chudak [4]. In the Euclidian plane $n$ points are randomly generated in the unit square $[0,1] \times[0,1]$. Each point simultaneously represents a facility and a client $(m=n)$, with $n=500,1000, \ldots, 3000$. The connection costs $c_{i j}$ are determined by the Euclidian distance. In each instance all the fixed costs $f_{i}$ are equal and calculated by $\sqrt{n} / l$ with $l=10,100$ or 1000 . All values are rounded up to 4 significant digits and made integer [23]. We use the label $n-l$ to name these instances.

The second set of UFL instances is called Koerkel-Ghosh. In these instances, the connection costs $c_{i j}$ are drawn uniformly at random from [1000,2000]. The fixed costs $f_{i}$ are drawn uniformly at random from $[100,200]$ in class 'a', from $[1000,2000]$ in class ' $b$ ' and from $[10000,20000]$ in class 'c'. Furthermore symmetric and asymmetric connection matrices are created. UflLib provides instances of the 3 largest sizes presented in [16] with $n=m=250,500$ and 750. Of the 90 instances proposed in the UflLib, we took 18 representative ones. We use the label gX00YZ-1 to name these instances, where X can be either ' $s$ ' or ' $a$ ' for the symmetric or asymmetric case respectively. $Y$ is equal to ' $n$ ' and $\mathrm{Z}$ is the class (a, b or c).

\subsection{CPLEX performance}

In Table 2 we report the results obtained with CPLEX 9.1 (default settings and 7200 seconds as CPU time limit). Barahona-Chudak instances: We observe that CPLEX has solved the first three instances. However, the remaining 15 instances have become too large, even to solve their LP relaxation. Koerkel-Gosh instances: CPLEX has given an integer solution and a lower bound in most of the cases. Cases gs00750a-1 and ga00750a-1 have become too large at the beginning of the B\&B process (CPLEX has been able to solve their LP relaxation and compute an integer solution). For the other 4 instances with 750 points, CPLEX has not even solved the LP relaxation within 7200 seconds.

\subsection{Dual solution quality}

In Table 3 we report the dual solution quality. We observe an important result: the semiLagrangian relaxation closes the duality gap (if convergence is reached). Column (3.a) reports the best known upper bound (UB) to the optimal primal cost. In the remaining 6 columns we report the results concerning the Lagrangian relaxation (LR) and the semi-Lagrangian relaxation (SLR) bounds. 
Table 1: Instance description: For the Barahona-Chudak instances, the fixed cost is the same for all facilities. For the Koerkel-Ghosh instances the fixed cost is randomly chosen for each facility according to a uniform distribution.

\begin{tabular}{lrrrrl}
\hline $\begin{array}{l}\text { Barahona-Chudak } \\
\text { instances }\end{array}$ & $\begin{array}{r}\text { Nb. of } \\
\text { clients }\end{array}$ & $\begin{array}{r}\text { Fix } \\
\text { cost }\end{array}$ & $\begin{array}{r}\text { Koerkel-Ghosh } \\
\text { instances }\end{array}$ & $\begin{array}{r}\text { Nb. of } \\
\text { clients }\end{array}$ & Fix \\
cost
\end{tabular}

In column (3.b) we have the LR lower bound (obtained with Proximal-ACCPM). In column (3.c) we give the LR duality gap (in fact we give an upper bound to the true duality gap since in some cases we do not know the true primal optimum but an upper bound). In the remaining 4 columns (3.d-g), we report the SLR lower bound information, obtained either with the dual ascent method o with Proximal-ACCPM. We also give the optimality gap of these dual values. Optimal SLR dual bounds have been written in boldface. In the remaining cases, the SLR procedure was stopped before reaching optimality because of the CPU time limit (7200 seconds).

\subsection{Primal solution quality}

In Table 4 we report the primal solution quality. Column (4.a) reports the best known lower bound. In column (4.b), we have the upper bounds (UB) to the UFL optima, reported in literature. In the first half of this column, we have the results for the Barahona-Chudak instances reported in [32], (they were obtained as the average of several runs). In the second half of this column, we have the results for the Koerkel-Ghosh instances as reported in UflLib (the results reported in [32] for these instances do not correspond to single instances but for groups of 5 instances). In columns (4.d) and (4.f) we have the UB obtained in the framework of the dual ascent method and Proximal-ACCPM, respectively.

In this section, the important result is that the SLR approach has been able to solve 18 instances of the 36 previously unsolved ones. These 18 instances have been solved by ProximalACCPM. On the other hand, the dual ascent method has been able to solve 16 UFL instances. 
Table 2: CPLEX performance: 'UB' stands for upper bound, 'LB' for lower bound. Larger instances have produced an 'Out of memory' either before CPLEX solved the LP relaxation or after ('Before LP' and 'After LP').

\begin{tabular}{|c|c|c|c|c|c|}
\hline Instance & UB & LB & B\&B gap (\%) & CPLEX time (sec.) & Out of memory \\
\hline $500-1000$ & 99169 & 99169 & 0.00 & 12 & \\
\hline $500-100$ & 326790 & 326790 & 0.00 & 4 & \\
\hline $500-10$ & 798577 & 798577 & 0.00 & 35 & \\
\hline $1000-1000$ & - & - & - & - & Before LP \\
\hline $1000-100$ & - & - & - & - & Before LP \\
\hline $1000-10$ & - & - & - & - & Before LP \\
\hline $1500-1000$ & - & - & - & - & Before LP \\
\hline $1500-100$ & - & - & - & - & Before LP \\
\hline $1500-10$ & - & - & - & - & Before LP \\
\hline $2000-1000$ & - & - & - & - & Before LP \\
\hline $2000-100$ & - & - & - & - & Before LP \\
\hline $2000-10$ & - & - & - & - & Before LP \\
\hline $2500-1000$ & - & - & - & - & Before LP \\
\hline $2500-100$ & - & - & - & - & Before LP \\
\hline $2500-10$ & - & - & - & - & Before LP \\
\hline $3000-1000$ & - & - & - & - & Before LP \\
\hline 3000-100 & - & - & - & - & Before LP \\
\hline $3000-10$ & - & - & - & - & Before LP \\
\hline gs00250a-1 & 257999 & 257803 & 0.08 & 7200 & \\
\hline gs00250b-1 & 276892 & 274324 & 0.94 & 7200 & \\
\hline gs00250c-1 & 332935 & 324142 & 2.71 & 7200 & \\
\hline gs00500a-1 & 512237 & 510446 & 0.35 & 7200 & \\
\hline gs00500b-1 & 654860 & 533027 & 22.86 & 7200 & \\
\hline gs00500c-1 & 746506 & 601986 & 24.01 & 7200 & \\
\hline gs00750a-1 & 785232 & 762565 & 2.97 & 5023 & After LP \\
\hline gs00750b-1 & - & - & - & 7200 & \\
\hline gs00750c-1 & - & - & - & 7200 & \\
\hline ga00250a-1 & 257991 & 257778 & 0.08 & 7200 & \\
\hline ga00250b-1 & 276556 & 274112 & 0.89 & 7200 & \\
\hline $\mathrm{ga} 00250 \mathrm{c}-1$ & 334735 & 324892 & 3.03 & 7200 & \\
\hline ga00500a-1 & 512071 & 510614 & 0.29 & 7200 & \\
\hline ga00500b-1 & 649226 & 533339 & 21.73 & 7200 & \\
\hline ga00500c-1 & 747626 & 602861 & 24.01 & 7200 & \\
\hline ga00750a-1 & 783973 & 762464 & 2.82 & 5707 & After LP \\
\hline ga00750b-1 & - & - & - & 7200 & \\
\hline ga00750c-1 & - & - & - & 7200 & \\
\hline
\end{tabular}


Table 3: Dual solution quality: 'UB' stands for upper bound, 'LB' for lower bound, 'PACCPM' for Proximal-ACCPM, 'DA' for dual ascent. Optimal lower bounds are in boldface.

\begin{tabular}{|c|c|c|c|c|c|c|c|}
\hline \multirow[t]{2}{*}{ Instance } & \multirow[t]{2}{*}{ UB } & \multicolumn{2}{|c|}{$\begin{array}{l}\text { Lagrangian } \\
\text { (PACCPM) }\end{array}$} & \multicolumn{2}{|c|}{$\begin{array}{l}\text { semi-Lagrangian } \\
\text { (Dual ascent) }\end{array}$} & \multicolumn{2}{|c|}{$\begin{array}{c}\text { semi-Lagrangian } \\
\text { (PACCPM) }\end{array}$} \\
\hline & & Bound & $\begin{array}{r}\text { Dual } \\
\text { Gap (\%) } \\
(3 . c)\end{array}$ & Bound & $\begin{array}{r}\text { Optimality } \\
\text { Gap (\%) } \\
(3 . \mathrm{e})\end{array}$ & Bound & $\begin{array}{r}\text { Optimality } \\
\text { Gap (\%) } \\
(3 . \mathrm{g})\end{array}$ \\
\hline $500-1000$ & 99169 & 99160 & 0.0091 & 99169 & 0 & 99169 & 0 \\
\hline $500-100$ & 326790 & 326790 & 0 & 326790 & 0 & 326790 & 0 \\
\hline 500-10 & 798577 & 798577 & 0 & 798577 & 0 & 798577 & 0 \\
\hline $1000-1000$ & 220560 & 220559 & 0.0003 & 220560 & 0 & 220560 & 0 \\
\hline $1000-100$ & 607878 & 607814 & 0.0105 & 607878 & 0 & 607878 & 0 \\
\hline $1000-10$ & 1434154 & 1433389 & 0.0534 & 1434154 & 0 & 1434154 & 0 \\
\hline $1500-1000$ & 334962 & 334944 & 0.0054 & 334962 & 0 & 334962 & 0 \\
\hline $1500-100$ & 866454 & 866453 & 0.0001 & 866454 & 0 & 866454 & 0 \\
\hline $1500-10$ & 2000801 & 1999284 & 0.0758 & 2000696 & 0.0052 & 2000801 & 0 \\
\hline $2000-1000$ & 437686 & 437678 & 0.0018 & 437686 & 0 & 437686 & 0 \\
\hline $2000-100$ & 1122748 & 1122746 & 0.0002 & 1122748 & 0 & 1122748 & 0 \\
\hline $2000-10$ & 2558118 & 2557753 & 0.0143 & 2558118 & 0 & 2558118 & 0 \\
\hline $2500-1000$ & 534405 & 534395 & 0019 & 534405 & 0 & 534405 & 0 \\
\hline $2500-100$ & 1347516 & 1347494 & 0.0016 & 1347516 & 0 & 1347516 & \\
\hline $2500-10$ & 3100225 & 3096856 & 1087 & 3097647 & 0.0831 & 3097647 & 0.0831 \\
\hline $3000-1000$ & 643463 & 643432 & .0048 & 643463 & 0 & 643463 & \\
\hline $3000-100$ & 1602335 & 1601652 & 0.0426 & 1602063 & 0.0170 & 1602120 & 0.0134 \\
\hline $3000-10$ & 3570766 & 3570752 & 0.0004 & 3570766 & 0 & 3570766 & 0 \\
\hline Partial average & 1200367.0 & 1199985 & 0.0184 & 1200203 & 0.0059 & 1200212 & 0.0054 \\
\hline gs00250a-1 & 257964 & 257639 & 1259 & 257899 & 0.0252 & 257964 & 0 \\
\hline gs00250b-1 & 276761 & 273693 & 1085 & 275363 & .5051 & 275574 & 0.4289 \\
\hline gs00250c-1 & 332935 & 322696 & 3.0753 & 329135 & 1414 & 330559 & 0.7137 \\
\hline gs00500a-1 & 511229 & 510408 & 0.1606 & 510408 & 0.1606 & 510408 & 0.1606 \\
\hline gs00500b-1 & 537931 & 533020 & 0.913 & 534029 & 0.7254 & 533477 & 0.8280 \\
\hline gs00500c-1 & 620041 & 601962 & 2.9158 & 607260 & 2.0613 & 609333 & 1.7270 \\
\hline gs00750a-1 & & 762562 & 0.1453 & 762562 & 0.1452 & 762562 & 0.1452 \\
\hline gs00750b-1 & 797026 & 790334 & 0.8396 & 790917 & 0.7665 & 790917 & 0.7665 \\
\hline gs00750c-1 & 900454 & 875340 & 2.789 & 879430 & 2.3348 & 879343 & 2.3445 \\
\hline ga00250a-1 & 257957 & 257618 & 0.1315 & 257882 & 0.0291 & 257957 & 0 \\
\hline ga00250b-1 & 276339 & 273296 & 1.1012 & 275080 & 0.4556 & 275200 & 0.4122 \\
\hline ga00250c-1 & 334135 & 322958 & 3.3451 & 329839 & 1.2857 & 331171 & 0.8871 \\
\hline ga00500a-1 & & 510587 & & 510587 & 0.1633 & 510587 & 0.1633 \\
\hline ga00500b-1 & 538060 & 533334 & 0.8784 & 534416 & 0.6772 & 533837 & 0.7849 \\
\hline ga00500c-1 & 621360 & 602852 & 2.9787 & 608518 & 2.0668 & 609975 & 1.8323 \\
\hline ga00750a-1 & 763576 & 762462 & 0.1459 & 762462 & 0.1459 & 762462 & 0.1459 \\
\hline ga00750b-1 & 796480 & 790112 & 0.7995 & 790631 & 0.7344 & 790630 & 0.7345 \\
\hline ga00750c-1 & 902026 & 875593 & 2.9304 & 880301 & 2.4085 & 879601 & 2.4861 \\
\hline Partial aver & 555520.4 & 547581 & 1.3637 & 549818 & 0.8795 & 550087 & 0.8089 \\
\hline Global average & 877943.7 & 873783 & 0.6910 & 875010 & 0.4427 & 875149 & 0.4071 \\
\hline
\end{tabular}


For the unsolved instances we have reported the upper bound obtained by the following simple heuristic which computes a primal feasible solution. At each iteration of the SLR method the oracle solution $y\left(u^{k}\right)$ proposes to open a set of facilities. To complete the solution, each client is assigned to its closest open facility. If the SLR method fails to converge, we take the best heuristic solution as the primal SLR solution. In general, the sophisticated heuristic used in [32] performs better than our simple primal heuristic. At the same time, the SLR procedure combined with this simple heuristic, has given better primal solutions than CPLEX.

\subsection{Instance reduction}

Very often, Lagrangian relaxation is used to decompose difficult problems. The decomposition induced by SLR is more coarse-grained than the Lagrangian relaxation one. Thus for example, in the UFL problem, after Lagrangian relaxation, one has one subproblem per facility. However, after SLR, one may have from several to only one subproblem. Furthermore, the number of subproblems usually is different for each SLR iteration. For this reason, we do not report the number of subproblems at each iteration, but its average. In Table 5, we have the average number of subproblems (ANS) per SLR iteration. More specifically, columns (5.a) and (5.b) report the ANS for the dual ascent method and for Proximal-ACCPM respectively. For example, in instance 500-1000 the ANS value is 108 and 84 for the dual ascent method and for Proximal.ACCPM, respectively. This means that the SLR method has managed to decomposed this instance. In other 30 instances (instance 500-100 for example) the SLR does not decompose the instance (ANS =1). Note that the decomposition is slightly better with the dual ascent method than with Proximal-ACCPM.

An important advantage of the SLR is that usually it drastically reduces the number of relevant variables (otherwise said, we can fix to 0 many variables). In column (5.c) we have the total number of $x_{i j}$ variables. For example, instance 500-1000 has $250000 x_{i j}$ variables, but, as we can see in column (5.d) only $0.3 \%$ are relevant in the SLR combined with the dual ascent method (the remaining $99.7 \%$ are fixed to 0 ). The number of relevant $x_{i j}$ variables in the case of Proximal-ACCPM is reported in (5.e). The analogous results for the $y_{i}$ variables can be found in columns (5.f-h). Note that the number of variables is different for each SLR iteration and therefore we give average figures corresponding to all the SLR iterations.

On average, in this test, the SLR only uses $2.5 \%$ of the $x_{i j}$ variables and $46 \%$ of the $y_{i}$ variables when we use the dual ascent algorithm (columns (5.d) and (5.g)). In the ProximalACCPM case we use a slightly higher number of variables (columns (5.e) and (5.h)).

\subsection{Performance}

Finally, in Table 6 we report the performance of the dual ascent method and Proximal-ACCPM in terms of the number of SLR iterations and CPU time. The CPU time limit is set as follows: we stop the SLR algorithm after the first completed SLR iteration that produces a cumulated CPU time above 7200 seconds. If that iteration, say the $k$ th one, goes beyond 10000 seconds, we stop the SLR procedure and report the results of the $(k-1)$ th iteration.

Proximal-ACCPM reduces by $60 \%$ the average number of dual ascent iterations. One would 
Table 4: Primal solution quality: 'SLR' stands for semi-Lagrangian relaxation, 'PACCPM' for Proximal-ACCPM, 'Opt.' for optimality, 'LB' for lower bound, 'UB' for upper bound and 'DA' for dual ascent. [32] obtains an upper bound by a heuristic method. Optimal costs for the UFL problem are in boldface.

\begin{tabular}{|c|c|c|c|c|c|c|c|}
\hline \multirow[t]{2}{*}{ Instance } & \multirow{2}{*}{$\begin{array}{l}\text { LB } \\
\quad(4 . a)\end{array}$} & \multicolumn{6}{|c|}{ UB } \\
\hline & & $\begin{array}{l}{[32]} \\
\text { (4.b) }\end{array}$ & $\begin{array}{r}\text { \% Opt. } \\
\text { (4.c) }\end{array}$ & $\begin{array}{r}\text { DA } \\
\text { (4.d) }\end{array}$ & $\begin{array}{r}\text { \% Opt. } \\
\text { (4.e) }\end{array}$ & $\begin{array}{r}\text { PACCPM } \\
(4 . f)\end{array}$ & $\begin{array}{r}\% \text { Opt. } \\
(4 . g)\end{array}$ \\
\hline $500-1000$ & 99169 & 99169.0 & 100 & 99169 & 100 & 99169 & 100 \\
\hline $500-100$ & 326790 & 326805.4 & 99.9953 & 326790 & 100 & 326790 & 100 \\
\hline $500-10$ & 798577 & 798577.0 & 100 & 798577 & 100 & 798577 & 100 \\
\hline $1000-1000$ & 220560 & 220560.9 & 99.9996 & 220560 & 100 & 220560 & 100 \\
\hline $1000-100$ & 607878 & 607880.4 & 99.9996 & 607878 & 100 & 607878 & 100 \\
\hline $1000-10$ & 1434154 & 1434185.4 & 99.9978 & 1434154 & 100 & 1434154 & 100 \\
\hline $1500-1000$ & 334962 & 334973.2 & 99.9967 & 334962 & 100 & 334962 & 100 \\
\hline $1500-100$ & 866454 & 866493.2 & 99.9955 & 866454 & 100 & 866454 & 100 \\
\hline $1500-10$ & 2000801 & 2001121.7 & 99.9840 & 2000801 & 100 & 2000801 & 100 \\
\hline $2000-1000$ & 437686 & 437690.7 & 99.9989 & 437686 & 100 & 437686 & 100 \\
\hline $2000-100$ & 1122748 & 1122861.9 & 99.9899 & 1122748 & 100 & 1122748 & 100 \\
\hline $2000-10$ & 2558118 & 2558120.8 & 99.9999 & 2558118 & 100 & 2558118 & 100 \\
\hline $2500-1000$ & 534405 & 534426.6 & 99.9960 & 534405 & 100 & 534405 & 100 \\
\hline $2500-100$ & 1347516 & 1347577.6 & 99.9954 & 1347516 & 100 & 1347516 & 100 \\
\hline $2500-10$ & 3097647 & 3100224.7 & 99.9168 & 3122045 & 99.2124 & 3122045 & 99.21237 \\
\hline $3000-1000$ & 643463 & 643541.8 & 99.9878 & 643463 & 100 & 643463 & 100 \\
\hline $3000-100$ & 1602120 & 1602530.9 & 99.9744 & 1602335 & 99.9866 & 1602397 & 99.98271 \\
\hline $3000-10$ & 3570766 & 3570818.8 & 99.9985 & 3570766 & 100 & 3570766 & 100 \\
\hline Partial average & 1200212 & 1200420.0 & 99.9903 & 1201579 & 99.9555 & 1201583 & 99.9553 \\
\hline gs00250a-1 & 257964 & 257964 & 100 & 258137 & 99.9329 & 257964 & 100 \\
\hline gs00250b-1 & 275574 & 276761 & 99.5693 & 279416 & 98.6058 & 278337 & 98.9974 \\
\hline gs00250c-1 & 330559 & 332935 & 99.2812 & 337270 & 97.9698 & 333617 & 99.0749 \\
\hline gs00500a-1 & 510408 & 511229 & 99.8392 & 513038 & 99.4847 & 513038 & 99.4847 \\
\hline gs00500b-1 & 534029 & 537931 & 99.2693 & 551716 & 96.6880 & 551716 & 96.6880 \\
\hline gs00500c-1 & 609333 & 620041 & 98.2427 & 641659 & 94.6949 & 641659 & 94.6949 \\
\hline gs00750a-1 & 762562 & 763671 & 99.8545 & 767269 & 99.3827 & 767269 & 99.3827 \\
\hline gs00750b-1 & 790917 & 797026 & 99.2275 & 810239 & 97.5569 & 810239 & 97.5569 \\
\hline gs00750c-1 & 879430 & 900454 & 97.6093 & 971616 & 89.5175 & 971616 & 89.5175 \\
\hline ga00250a-1 & 257957 & 257969 & 99.9953 & 258363 & 99.8426 & 257957 & 100 \\
\hline ga00250b-1 & 275200 & 276339 & 99.5861 & 280695 & 98.0033 & 278442 & 98.8219 \\
\hline ga00250c-1 & 331171 & 334135 & 99.1050 & 346296 & 95.4329 & 337398 & 98.1197 \\
\hline ga00500a-1 & 510587 & 511422 & 99.8365 & 513673 & 99.3956 & 513673 & 99.3956 \\
\hline ga00500b-1 & 534416 & 538060 & 99.3181 & 546253 & 97.7851 & 546253 & 97.7851 \\
\hline ga00500c-1 & 609975 & 621360 & 98.1335 & 670597 & 90.0616 & 643140 & 94.5629 \\
\hline ga00750a-1 & 762462 & 763576 & 99.8539 & 767965 & 99.2783 & 767965 & 99.2783 \\
\hline ga00750b-1 & 790631 & 796480 & 99.2602 & 808105 & 97.7899 & 808105 & 97.7899 \\
\hline ga00750c-1 & 880301 & 902026 & 97.5321 & 1000972 & 86.2921 & 1008497 & 85.4373 \\
\hline Partial average & 550193 & 555521 & 99.1952 & 573516 & 96.5397 & 571494 & 97.0326 \\
\hline Global average & 875202 & 877970.5 & 99.5928 & 887547 & 98.2476 & 886538 & 98.4940 \\
\hline
\end{tabular}


Table 5: Semi-Lagrangian reduction and decomposition: 'ANS' stands for average number of subproblems per SLR iteration, 'DA' for dual ascent, 'PACCPM' for proximal-ACCPM, 'ANX' for average number of relevant $x_{i j}$ variables per SLR iteration (in \%) and 'ANY' for average number of relevant $y_{i}$ variables per SLR iteration (in \%).

\begin{tabular}{|c|c|c|c|c|c|c|c|c|}
\hline \multirow[t]{2}{*}{ Instance } & \multicolumn{2}{|c|}{ ANS } & \multirow{2}{*}{$\begin{array}{c}\text { Total } \\
\text { Nb. of } x_{i j} \\
(5 . c)\end{array}$} & \multicolumn{2}{|c|}{$\operatorname{ANX}(\%)$} & \multirow{2}{*}{$\begin{array}{c}\text { Total } \\
\text { Nb. of } y_{i} \\
(5 . f)\end{array}$} & \multicolumn{2}{|c|}{ ANY (\%) } \\
\hline & $\begin{array}{r}\text { DA } \\
(5 . a)\end{array}$ & $\begin{array}{r}\text { PACCPM } \\
(5 . b)\end{array}$ & & $\begin{array}{r}\text { DA } \\
(5 . d)\end{array}$ & $\begin{array}{r}\text { PACCPM } \\
(5 . \mathrm{e})\end{array}$ & & $\begin{array}{r}\text { DA } \\
(5 . \mathrm{g})\end{array}$ & $\begin{array}{r}\text { PACCPM } \\
(5 . h)\end{array}$ \\
\hline $500-1000$ & 108 & 84 & 250000 & 0.3 & 0.5 & 500 & 56 & 98 \\
\hline $500-100$ & 1 & 1 & 250000 & 1.2 & 1.2 & 500 & 66 & 66 \\
\hline $500-10$ & 1 & 1 & 250000 & 2.8 & 2.8 & 500 & 35 & 35 \\
\hline $1000-1000$ & 106 & 29 & 1000000 & 0.3 & 0.3 & 1000 & 89 & 94 \\
\hline $1000-100$ & 1 & 1 & 1000000 & 0.8 & 0.9 & 1000 & 55 & 61 \\
\hline $1000-10$ & 1 & 1 & 1000000 & 2.6 & 3.4 & 1000 & 39 & 51 \\
\hline $1500-1000$ & 11 & 7 & 2250000 & 0.3 & 0.3 & 1500 & 86 & 87 \\
\hline $1500-100$ & 1 & 1 & 2250000 & 0.6 & 0.6 & 1500 & 50 & 50 \\
\hline $1500-10$ & 1 & 1 & 2250000 & 1.8 & 2.6 & 1500 & 30 & 44 \\
\hline $2000-1000$ & 2 & 2 & 4000000 & 0.2 & 0.2 & 2000 & 82 & 82 \\
\hline $2000-100$ & 1 & 1 & 4000000 & 0.6 & 0.6 & 2000 & 50 & 50 \\
\hline $2000-10$ & 1 & 1 & 4000000 & 1.2 & 2.2 & 2000 & 25 & 42 \\
\hline $2500-1000$ & 3 & 3 & 6250000 & 0.2 & 0.2 & 2500 & 80 & 80 \\
\hline $2500-100$ & 1 & 1 & 6250000 & 0.5 & 0.6 & 2500 & 46 & 58 \\
\hline $2500-10$ & 1 & 1 & 6250000 & 1.7 & 1.7 & 2500 & 35 & 35 \\
\hline $3000-1000$ & 2 & 2 & 9000000 & 0.2 & 0.2 & 3000 & 78 & 78 \\
\hline $3000-100$ & 1 & 1 & 9000000 & 0.5 & 0.6 & 3000 & 53 & 56 \\
\hline $3000-10$ & 1 & 1 & 9000000 & 1.0 & 1.0 & 3000 & 23 & 23 \\
\hline Partial average & 14 & 8 & 3791667 & 0.9 & 1.1 & 1750 & 54 & 61 \\
\hline gs00250a-1 & 1 & 1 & 62500 & 2.4 & 4.6 & 250 & 60 & 87 \\
\hline gs00250b-1 & 1 & 1 & 62500 & 5.6 & 6.3 & 250 & 50 & 54 \\
\hline gs00250c-1 & 1 & 1 & 62500 & 14.4 & 18.4 & 250 & 43 & 52 \\
\hline gs00500a-1 & 1 & 1 & 250000 & 1.3 & 1.3 & 500 & 48 & 48 \\
\hline gs00500b-1 & 1 & 1 & 250000 & 1.8 & 2.2 & 500 & 24 & 30 \\
\hline gs00500c-1 & 1 & 1 & 250000 & 5.1 & 6.1 & 500 & 23 & 27 \\
\hline gs00750a-1 & 1 & 1 & 562500 & 1.0 & 1.0 & 750 & 48 & 48 \\
\hline gs00750b-1 & 1 & 1 & 562500 & 1.6 & 1.6 & 750 & 26 & 26 \\
\hline gs00750c-1 & 1 & 1 & 562500 & 3.0 & 3.0 & 750 & 17 & 17 \\
\hline ga00250a-1 & 1 & 1 & 62500 & 2.5 & 4.3 & 250 & 62 & 85 \\
\hline ga00250b-1 & 1 & 1 & 62500 & 5.5 & 5.9 & 250 & 48 & 50 \\
\hline ga00250c-1 & 1 & 1 & 62500 & 13.5 & 16.9 & 250 & 40 & 47 \\
\hline ga00500a-1 & 1 & 1 & 250000 & 1.4 & 2.4 & 500 & 53 & 31 \\
\hline ga00500b-1 & 1 & 1 & 250000 & 2.4 & 1.4 & 500 & 31 & 24 \\
\hline ga00500c-1 & 1 & 1 & 250000 & 5.2 & 6.3 & 500 & 23 & 30 \\
\hline ga00750a-1 & 1 & 1 & 562500 & 1.0 & 1.0 & 750 & 44 & 44 \\
\hline ga00750b-1 & 1 & 1 & 562500 & 1.3 & 1.5 & 750 & 23 & 24 \\
\hline ga00750c-1 & 1 & 1 & 562500 & 2.9 & 2.7 & 750 & 16 & 15 \\
\hline Partial average & 1 & 1 & 291667 & 4.0 & 4.8 & 500 & 38 & 41 \\
\hline Global average & 7 & 4 & 2041667 & 2.5 & 3.0 & 1125 & 46 & 51 \\
\hline
\end{tabular}




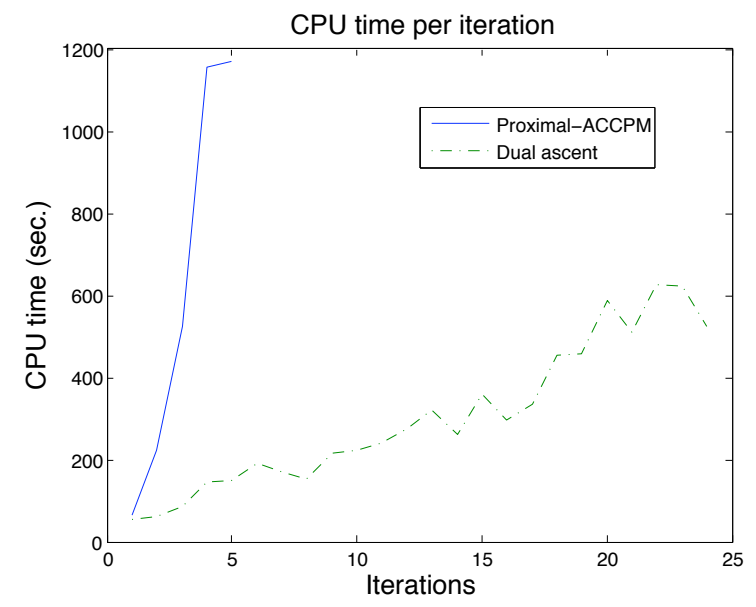

Figure 1: CPU time per iteration for instance 1500-10.

expect a similar reduction in the CPU time, instead of the mentioned 35\%. The reason for this mismatch, as we can see in (6.e) and (6.f), is that the average CPU time per SLR iteration is greater for Proximal-ACCPM than for the dual ascent algorithm. This is because ProximalACCPM perform a faster increase of the Lagrange multiplier values, which produces a faster increase in the number of relevant variables, as observed in Table 5. This produces harder (CPU time consuming) Oracle 2's. (See Fig. 1 and Fig. 2.)

As usual, a heuristic method reduces the CPU time of an exact method at the price of no information about the solution quality. If we compare the best SLR results (column (6.f)) versus the results reported in [32] (column (6.g)) we observe that: for the Barahona-Chudak instances, the SLR CPU time is very competitive, since at the price of an extra $64 \%$ of CPU time, we solve up to optimality 16 instances. For the 2 remaining instances we can evaluate its quality by the SLR lower bound. For the Koerkel-Ghosh instances SLR is less competitive (quality solution and CPU time) compared to [32]. However, for these instances and by using the SLR bounds we can determine for the first time that, on average, the [32] solutions are at least $99.18 \%$ optimal (Table 4).

\section{Conclusions}

The contributions of this paper are twofold: empirical and theoretical.

Empirical contribution:We have shown by example that the performance of a general MIP solver, as CPLEX, can be enhanced by combining it with the semi-Lagrangian relaxation (SLR) approach. In our computational experiments, CPLEX solved 3 out of 36 Uncapacitated Facility Location (UFL) unsolved instances from the UflLib. In contrast, by using the SLR together with two standard optimization tools, CPLEX and Proximal-ACCPM, we solved 18 instances. For the remaining 18 still unsolved UFL instances, we have improved the best known lower bound and confirmed, for the first time, that the Hybrid Multistart heuristic of [32] provides near optimal solutions (over 99\% optimal). The reason for this good result is that, the SLR drastically reduced the number of UFL relevant variables. Roughly speaking, Onthe average 
Table 6: Performance: 'PACCPM' stands for proximal-ACCPM and 'DA' for dual ascent. [32] uses an heuristic method.

\begin{tabular}{|c|c|c|c|c|c|c|c|}
\hline \multirow[t]{3}{*}{ Instance } & \multicolumn{3}{|c|}{ Nb. of completed iterations } & \multicolumn{4}{|c|}{ Total CPU time (sec.) } \\
\hline & \multirow{2}{*}{$\begin{array}{c}\text { LR } \\
\text { PACCPM } \\
(6 . a)\end{array}$} & \multicolumn{2}{|c|}{ SLR } & \multirow{2}{*}{$\begin{array}{c}\text { LR } \\
\text { PACCPM } \\
(6 . d)\end{array}$} & \multicolumn{2}{|c|}{$\mathrm{LR}+\mathrm{SLR}$} & \multirow{2}{*}{$\begin{array}{l}{[32]} \\
(6 . g)\end{array}$} \\
\hline & & $\begin{array}{r}\text { DA } \\
(6 . b)\end{array}$ & $\begin{array}{r}\text { PACCPM } \\
\text { (6.c) }\end{array}$ & & $\begin{array}{r}\text { DA } \\
(6 . e)\end{array}$ & $\begin{array}{r}\text { PACCPM } \\
\text { (6.f) }\end{array}$ & \\
\hline $500-1000$ & 128 & 1 & 1 & 2.7 & 3 & 4 & 33 \\
\hline $500-100$ & 100 & 1 & 1 & 1.9 & 2 & 2 & 33 \\
\hline $500-10$ & 177 & 1 & 1 & 4.1 & 5 & 5 & 24 \\
\hline $1000-1000$ & 121 & 1 & 1 & 3.5 & 6 & 6 & 174 \\
\hline $1000-100$ & 276 & 2 & 2 & 11.8 & 16 & 19 & 149 \\
\hline $1000-10$ & 535 & 29 & 5 & 46.1 & 4024 & 1061 & 142 \\
\hline $1500-1000$ & 128 & 1 & 1 & 5.0 & 9 & 10 & 348 \\
\hline $1500-100$ & 311 & 1 & 1 & 19.0 & 22 & 22 & 379 \\
\hline $1500-10$ & 327 & 24 & 5 & 19.3 & 7438 & 3156 & 387 \\
\hline $2000-1000$ & 137 & 1 & 1 & 7.0 & 12 & 12 & 718 \\
\hline 2000-100 & 245 & 1 & 1 & 16.7 & 23 & 23 & 651 \\
\hline $2000-10$ & 408 & 40 & 4 & 37.7 & 2256 & 661 & 760 \\
\hline $2500-1000$ & 136 & 1 & 1 & 9.4 & 18 & 18 & 1420 \\
\hline $2500-100$ & 342 & 2 & 2 & 36.1 & 55 & 89 & 1128 \\
\hline $2500-10$ & 754 & 1 & 1 & 177.7 & 8214 & 8214 & 1309 \\
\hline $3000-1000$ & 160 & 5 & 3 & 14.0 & 76 & 63 & 1621 \\
\hline $3000-100$ & 427 & 10 & 8 & 64.1 & 7355 & 8428 & 1978 \\
\hline $3000-10$ & 413 & 1 & 1 & 60.5 & 90 & 90 & 2081 \\
\hline Partial average & 285 & 7 & 2 & 29.8 & 1646 & 1216 & 741 \\
\hline gs00250a-1 & 209 & 11 & 5 & 7.1 & 8173 & 5765 & 6 \\
\hline gs00250b-1 & 165 & 11 & 3 & 2.9 & 9145 & 3845 & 8 \\
\hline gs00250c-1 & 252 & 37 & 13 & 5.2 & 7831 & 7929 & 7 \\
\hline gs00500a-1 & 195 & 0 & 0 & 9.5 & 10 & 10 & 40 \\
\hline gs00500b-1 & 191 & 2 & 1 & 10.2 & 2771 & 515 & 52 \\
\hline gs00500c-1 & 143 & 14 & 5 & 7.1 & 7241 & 7573 & 57 \\
\hline gs00750a-1 & 192 & 0 & 0 & 12.9 & 13 & 13 & 118 \\
\hline gs00750b-1 & 143 & 1 & 1 & 11.3 & 5436 & 5234 & 127 \\
\hline gs00750c-1 & 140 & 8 & 2 & 10.7 & 7274 & 1868 & 137 \\
\hline ga00250a-1 & 177 & 9 & 4 & 5.9 & 7389 & 6567 & 5 \\
\hline ga00250b-1 & 229 & 12 & 3 & 4.6 & 7283 & 2223 & 8 \\
\hline ga00250c-1 & 156 & 36 & 13 & 2.5 & 7675 & 7804 & 8 \\
\hline ga00500a-1 & 233 & 0 & 0 & 9.9 & 10 & 10 & 44 \\
\hline ga00500b-1 & 229 & 3 & 1 & 4.6 & 8899 & 478 & 53 \\
\hline ga00500c-1 & 150 & 16 & 5 & 5.3 & 7936 & 6604 & 51 \\
\hline ga00750a-1 & 199 & 0 & 0 & 14.1 & 14 & 14 & 113 \\
\hline ga00750b-1 & 137 & 1 & 1 & 10.6 & 3134 & 3181 & 126 \\
\hline ga00750c-1 & 126 & 10 & 2 & 7.7 & 9018 & 1554 & 130 \\
\hline Partial averag & 181 & 10 & 3 & 7.9 & 5514 & 3399 & 61 \\
\hline Global average & 233 & 8 & 3 & 18.8 & 3580 & 2307 & 401 \\
\hline
\end{tabular}




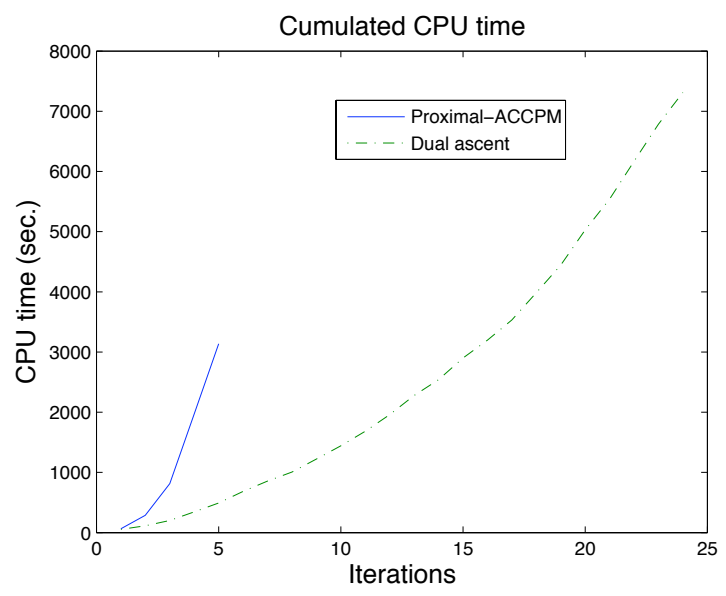

Figure 2: Cumulated CPU time for the instance 1500-10.

number of relevant $x_{i j}$ variables was reduced to $3 \%$ and the average number of relevant $y_{i}$ variables, was reduced to $50 \%$.

Also from an empirical point of view, we have compared two dual optimization methods: Proximal-ACCPM and a dual ascent method. Proximal-ACCPM has shown a better performance than the dual ascent: it has produced similar and sometimes better solutions with a CPU time reduction of $35 \%$. Within the 2 hours of CPU time limit, Proximal-ACCPM and the dual ascent method have fully solved 18 and 16 UFL instances, respectively (from a pool of 36 unsolved instances). The advantage of the dual ascent method is its extreme simplicity compared to Proximal-ACCPM.

Theoretical contribution: From a theoretical point of view, this paper has proposed an extension of the Koerkel dual multi-ascent method to solve the SLR dual problem and we have proved (finite) convergence. Furthermore, We have studied the theoretical properties of the SLR dual problem in the UFL case. We have shown that for the UFL problem we can restrict our dual search to a box whose definition depends on the problem costs. This property has not shown to be very useful for the dual ascent method. In contrast, in the case of Proximal-ACCPM the explicit use of this box has slightly improved the dual search.

\section{Acknowledgments}

We are thankful: To Mato Baotic (Swiss Federal Institute of Technology) for its Matlab/Cplex interface [3], to Martin Hoefer, (Max Planck Institut Informatik) for the UFL data from the UflLib [23], and to the anonymous referees for their constructive comments and suggestions.

\section{References}

[1] P. Avella, A. Sassano, and I. Vasil'ev. Computational study of large-scale p-median problems. Mathematical Programming, 109(1):89-114, 2007.

[2] F. Babonneau, C. Beltran, A. B. Haurie, C. Tadonki, and J.-Ph. Vial. Proximal-ACCPM: a versatile oracle based optimization method, pages 200-224. Advances in Computational Economics, Finance and Management Science, volume of books series on Advances 
on Computational Management Science, Kontoghiorghes, E. J. and Gatu, C. (Editors). Springer, 2006.

[3] M. Baotic. Matlab interface for cplex, http://control.ee.ethz.ch/ hybrid/cplexint.php 2004.

[4] F. Barahona and F. Chudak. Near-optimal solutions to large scale facility location problems technical report. Technical Report RC21606, IBM Watson Research Center, 1999.

[5] C. Beltran, C. Tadonki, and J.-Ph. Vial. Solving the p-median problem with a semiLagrangian relaxation. Computational Optimization and Applications, 35(2):239-260, 2006.

[6] C. Beltran-Royo. A conjugate Rosen's gradient projection method with global line search for piecewise linear concave optimization. European Journal of Operational Research, 182(2):536-551, 2007.

[7] O. Briant and D. Naddef. The optimal diversity management problem. Operations Research, 52(4), 2004.

[8] L. Canovas, L Landete, and A. Marin. On the facets of the simple plant location packing polytope. Discrete Applied Mathematics, 124:27-53, 2002.

[9] D. Ch. Cho, E. L. Johnson, M. W. Padberg, and M. R. Rao. On the uncapacitated facility location problem I: Valid inequalities and facets. Mathematics of Operations Reserach, 8(4):590-612, 1983.

[10] D. Ch. Cho, M. W. Padberg, and M. R. Rao. On the uncapacitated facility location problem II: Facets and lifting theorems. Mathematics of Operations Reserach, 8(4):590-612, 1983.

[11] A. R. Conn and G. Cornuéjols. A projection method for the uncapacitated facility location problem. Mathematical programming, 46:373-398, 1990.

[12] A. de Silva and D. Abramson. A parallel interior point method and its application to facility location problems. Computational Optimization and Applications, 90(3):249-273, 1998.

[13] O. du Merle and J.-Ph. Vial. Proximal-ACCPM, a cutting plane method for column generation and Lagrangian relaxation: application to the p-median problem. Technical report, Logilab, HEC, University of Geneva, 2002.

[14] D. Erlenkotter. A dual-based procedure for uncapacitated facility location. Operations Research, 26:992-1009, 1978.

[15] L.-L. Gao and E. P. Robinson. Uncapacitated facility location: General solution procedure and computational experience. European Journal of Operational Research, 76(3):410 417, 1994.

[16] D. Ghosh. Neighborhood search heuristics for the uncapacitated facility location problem. European Journal of Operational Research, 150:150-162, 2003.

[17] J.-L. Goffin, A. Haurie, and J.-Ph. Vial. Decomposition and nondifferentiable optimization with the projective algorithm. Management Science, 37:284-302, 1992. 
[18] J.-L. Goffin and J.-Ph. Vial. Convex nondifferentiable optimization: A survey focussed on the analytic center cutting plane method. Optimization Methods and Software, 17(805867), 2002.

[19] M. Guignard. A Lagrangean dual ascent algorithm for simple plant location problems. European Journal of Operational Research, 35:193-200, 1988.

[20] M. Guignard. Lagrangian relaxation. TOP (Journal of the "Spanish Society of Statistics and Operations Research"), 11(2):151-228, 2003.

[21] J. B. Hiriart-Urruty and C. Lemaréchal. Convex Analysis and Minimization Algorithms, volume I and II. Springer-Verlag, Berlin, 1996.

[22] J.-B. Hiriart-Urruty and C. Lemaréchal. Fundamentals of convex analysis. Springer, 2000.

[23] M. Hoefer. Ufllib, 2006. http://www.mpi-inf.mpg.de/departments/d1/projects/benchmarks/UflLib/.

[24] J. Janacek and L. Buzna. An acceleration of erlenkotter-koerkels algorithms for the uncapacitated facility location problem. Annals of Operations Research, 164:97-109, 2008.

[25] J. E. Kelley. The cutting-plane method for solving convex programs. Journal of the SIAM, 8:703-712, 1960.

[26] Y. Kochetov and D. Ivanenko. Computationally difficult instances for the uncapacitated facility location problem. In T. Ibaraki, K. Nonobe, and M. Yagiura, editors, Metaheuristics: progress as real solvers. Springer, 2005.

[27] M. Koerkel. On the exact solution of large-scale simple plant location problems. European Journal of Operational Research, 39(2):157-173, 1989.

[28] M. Landete and A. Marin. New facets for the two-stage uncapacitated facility location polytope. Computational Optimization and Applications, In press, 2009.

[29] P. Mirchandani and R. Francis. Discrete location theory. John Wiley and Sons, 1990.

[30] N. Mladenovic, J. Brimberg, and P. Hansen. A note on duality gap in the simple plant location. European Journal of Operational Research, 174(1):11-22, 2006.

[31] G. L. Nemhauser and L. A. Wolsey. Integer and Combinatorial Optimization. John Wiley and Sons, 1988.

[32] M. G. G. Resende and R. F. Werneck. A hybrid multistart heuristic for the uncapacitated facility location problem. European Journal of Operational Research, 174(1):54-68, 2006.

[33] M. Sun. Solving uncapacitated facility location problems using tabu search. Computers and Operations Research, 33:2563-2589, 2006. 